treated with a pharmaceutical product which does not necessarily have a causal relationship with this treatment". AEs were subdivided in non-infectious, infectious, infusion and hypersensitivity reactions. Infusion and hypersensitivity reactions were defined as transient AEs related to belimumab occurring within 6 hours and 6-48 hours after drug administration, respectively. AEs was defined as severe (SAE) in case of hospitalization and/or death and/or life-threatening manifestations. Infections were considered severe in case of hospitalization and/or intravenous antibiotic use and/or death. Infusion and hypersensitivity reactions were considered severe when intensive care unit support was required. As baseline predictors of discontinuation the following variables were analyzed: gender, age, age at SLE onset, disease duration, disease activity pattern (relapsing remitting or chronic active), SLEDAI-2K $\geq 10$, prednisone $>7.5 \mathrm{mg} /$ day, concomitant immunosuppressant, antimalarial drug use, number and type of comorbidities, number of previous organ involvement, type of major involvement and number of flares in the 12 months before belimumab initiation. Data were analyzed using the SPSS (version 23.0, Chicago, IL) software.

Results: A total of 453 EAs were recorded in 132 patients after a mean follow-up period of $17.5 \pm 10.6$ months (range 3-36): 443 (97.8\%) were non severe and 10 (2.2\%) SAEs (Table 1). No deaths and severe infusion/hypersensitivity reactions occurred. Belimumab discontinuation was observed in 58 patients $(30.8 \%)$ after $10.4 \pm 7.5$ months of follow-up (Table 2). RR was $91.5 \%$ at 6 months, $81.4 \%$ at 12 months, $72.9 \%$ at 18 months, $72.4 \%$ at 24 months, $69.7 \%$ at 30 months and $69.2 \%$ at 36 months. No associations were found between baseline variables and drug discontinuation.

Table 1. AEs observed in 188 patients with refractory SLE treated with belimumab Ptswith AEs (N) Pts with AEs (\%) AEs (N) AEs (\%

\begin{tabular}{lcccc} 
Non severe AEs & 129 & 68.6 & 443 & 97.8 \\
Infectious AEs & 101 & 53.7 & 273 & 60.3 \\
Non infectious AEs & 57 & 30.3 & 84 & 18.5 \\
Hypersensitivityreactions & 26 & 13.8 & 81 & 17.9 \\
Infusion reactions & 4 & 2.1 & 5 & 1.1 \\
SAEs & 9 & 4.8 & 10 & 2.2 \\
Infectious SAEs & 1 & 0.5 & 1 & 0.2 \\
Non infectious SEAs & 8 & 4.3 & 9 & 2.0 \\
\hline Total & 132 & 70.2 & 453 & $\mathbf{1 0 0 . 0}$ \\
\hline
\end{tabular}

Pts: patients; AEs: acherse events; SAEs: severe acherse events.

Table 2. Reasons of discontinuation of belimumab

\begin{tabular}{lcc} 
& Pts (N) & Pts (\%) \\
AEs & 22 & 37.9 \\
Non-responder (articular/renal cutaneous haematological) & 16 & 27.6 \\
Pregnancy & 8 & 13.8 \\
Flare (renal/articular) & 6 & 10.3 \\
Lost of follow-up & 4 & 6.9 \\
Remission & 2 & 3.4 \\
Total & $\mathbf{5 8}$ & $\mathbf{1 0 0 . 0}$ \\
\hline Pts: potients. & &
\end{tabular}

Conclusions: Belimumab demonstrated a good safety profile with a low rate of SAEs. Discontinuation occurred in less than $1 / 3$ of subjects with a low rate of discontinuation due to AEs.

Disclosure of Interest: None declared

DOI: 10.1136/annrheumdis-2017-eular.6088

\section{AB0457 LONG-TERM EFFECTIVENESS OF TREATMENTS FOR NEUROPSYCHIATRIC MANIFESTATIONS OF SYSTEMIC LUPUS ERYTHEMATOSUS}

\section{L.D. Fajardo Hermosillo. Rheumatology, IMSS, Guadalajara, Mexico}

Background: Neuropsychiatric involvement of systemic lupus erythematosus (NPSLE) is one of the most deleterious complications of the disease, leading to great decrease of quality of life and poor prognosis [1]. Factors such as activity of disease have been associated NPSLE [1,2]. The current treatment of NPSLE remains mostly empiric, requiring high-dose corticosteroids and extensive immunosuppressive therapy $[3,4]$. Short-term efficacy of treatments in NPSLE have been evaluated by several studies, however few studies have been performed to evaluate long-term efficacy of these drugs [4].

Objectives: To compare the long-term effectiveness among drugs and associated factors in the treatment of NPSLE.

Methods: 209 patients ( $\geq 14$ years) from a Mexican cohort from 2011 to 2017 were examined. SLE and NPSLE cases fulfilled 1997 ACR and 1999 ACR criteria respectively. Demographic factors, comorbidities and pharmacologic treatments were reviewed for patients with NPSLE. Variables were studied by bivariate, multivariate and survival analyses.

Results: Of 209 SLE patients, 37 (17.7\%) had NPSLE; of them 32 were women. The mean age [standard deviation (SD)] was 38.8 (14.6) years. The mean of time at onset of SLE (SD) was 6.2 (5.4) years. SLEDAI and SLICC mean (SD) were $22.1(10.2)$ and $1.83(1.2)$ separately. Diffuse and focal manifestations of NPSLE were presented in 20 and 17 cases. Central and peripheral nervous system events of NPSLE were described in 34 and 3 of patients. The NPSLE manifestations more prevalent were cerebrovascular disease $(n=15)$, psychosis $(n=8)$ and seizure disorders $(n=5)$. A total of 112 cycles of treatment were analyzed [prednisone (PDN) $n=30$, intravenous (iv) methylprednisolone (MTP) $n=23$, iv cyclophosphamide (CYC) $n=13$, azathioprine (AZA) $n=12$, mycophenolate mofetil (MMF) $n=11$, rituximab (RTX) $n=10$, hydroxichloroquine (HCQ) $n=9$, plasmapheresis (PPH) $n=2$ and iv immunoglobulin (IVIg) $n=2$ ]. Cognitive dysfunction was more associated to higher SLE activity, damage and mortality. Also, psychosis was more associated to receive higher doses of oral PDN or iv MTP, to employ iv CYC and to be linked with renal SLE disease activity. On the other hand, cerebral vascular disease was more associated to receive $\mathrm{HCQ}$ and to have antiphospholipid antibodies. The main cause to therapy discontinuation was inefficacy and was more common in patients treated with iv CYC. Corticosteroids survival in months was higher in-group PDN $<15$ $\mathrm{mg} / \mathrm{d}(97.89 \pm 16.4$; IC95\% 65.8-130). AZA survival in months $(90 \pm 30.8$; IC95\% 29.6-150) was higher than others treatments for NPSLE and was more associated to use IVIg (OR 10.8, 95\% Cl 1.04-111, $\mathrm{p}=0.04$ ). When the first therapy was failed, the drug as second therapy used with higher survival in months was MMF

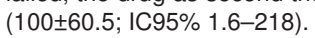

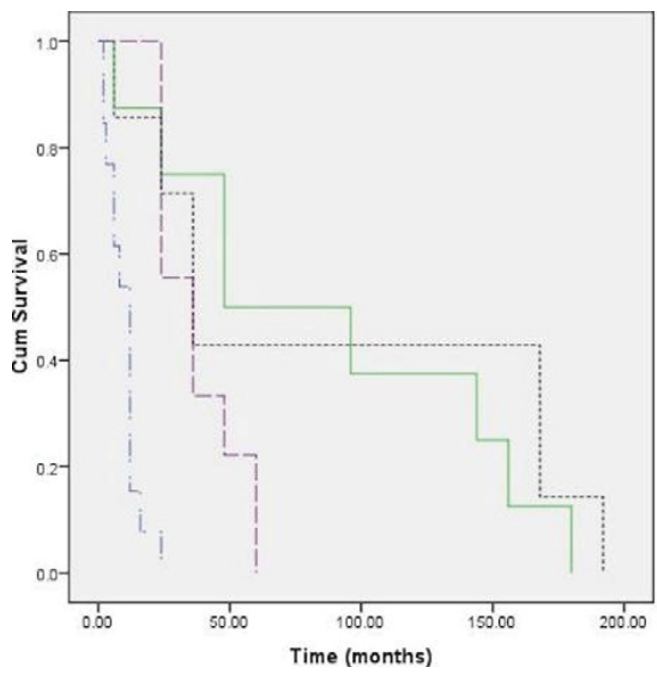

Treatments $\sim$ NAZA $\rightarrow$ TMUF

Conclusions: This study suggests that patients with diffuse manifestations and central nervous system involvement in NPSLE presented more activity, damage and mortality and therefore used more PDN, MTP and CYC. Among the treatments as first therapy for NPSLE with the better long-term efficacy was AZA and when it failed, MMF remained as better second therapy.

\section{References:}

[1] Arthritis Rheum 1999;42:599-608.

[2] Semin Arhtritis Rheum 2011;41;1-11.

[3] Ann Rheum Dis 2010;69(3):529-535.

[4] CNS DRugs 2011;25(9):721-736.

Disclosure of Interest: None declared

DOI: 10.1136/annrheumdis-2017-eular.2815

\section{AB0458 B CELLS DEPLETION FOR THE TREATMENT OF SYSTEMIC AUTOIMMUNE DISEASES}

M.L. Velloso Feijoo, S. Rodriguez Montero, N. Plaza Aulestia, J.L. Marenco de la Fuente. Rheumatology Unit, Valme University Hospital, Seville, Spain

Background: Systemic autoimmune diseases (SAD) have traditionally been treated with steroids and immunosuppressants, but not all patients respond to these measures.

Rituximab (RTX) has been used in several SAD with favorable results, but there are only a few reports of isolated clinical experiences, with a very small number of patients.

Data about use of this drug under conditions of daily clinical practice may be relevant.

Objectives: To describe the characteristics of patients with SAD who are candidates for treatment with RTX.

To evaluate the response to this drug in the different SAD.

Methods: Demographic data and disease, treatment and response variables were included.

We use the EULAR definitions of partial response (improvement of at least $50 \%$ of the main manifestations of the disease) and complete response (disappearance of the manifestations of the disease), because of the heterogeneity of the SAD and their multiple manifestations.

Results: We included 53 patients, 90.6\% were women. The mean age at diagnosis was $31.42 \pm 14.33$ years; and the median duration of disease at the onset of RTX $1.99(0-7.5)$ years. Patients received a median of 2 cycles (1-3 min 